\title{
Visualization of heat flow in a vertical channel with fully developed mixed convection ${ }^{2}$
}

\author{
Hasan Celik, Moghtada Mobedi * \\ Mechanical Engineering Department, Izmir Institute of Technology, Urla 35430 Izmir, Turkey
}

\section{A R T I C L E I N F O}

Available online 27 June 2012

\section{Keywords:}

Mixed convection

Porous medium

Heatfunction

Heatline

\begin{abstract}
A B S T R A C T
A study on visualization of heat flow in three channels with laminar fully developed mixed convection heat transfer is performed. The first channel is filled with completely pure fluid; the second one is completely filled with fluid saturated porous medium. A porous layer exists in the half of the third channel while another half is filled with pure fluid. The velocity, temperature and heat transport fields are obtained both by using analytical and numerical methods. Analytical expression for heat transport field is obtained and presented. The heatline patterns are plotted for different values of $\mathrm{Gr} / \mathrm{Re}$, thermal conductivity ratio, Peclet and Darcy numbers. It is found that the path of heat flow in the channel strongly depends on Peclet number. For low Peclet numbers (i.e., $P e=0.01$ ), the path of heat flow is independent of $\mathrm{Gr} / \mathrm{Re}$ and Darcy numbers. However, for high Peclet numbers (i.e., $\mathrm{Pe}=5$ ), the ratio of $\mathrm{Gr} / \mathrm{Re}$, Darcy number and thermal conductivity ratio influence heatline patterns, considerably. For the channels with high Peclet number (i.e., Pe $=5$ ), a downward heat flow is observed when a reverse flow exits.
\end{abstract}

(C) 2012 Elsevier Ltd. All rights reserved.

\section{Introduction}

Isotherms and streamlines are widely used in convective heat transfer studies in order to describe heat and fluid flow in a domain. However, understanding heat flow direction is not easy by using isotherms and streamlines. That is why, heatline technique was proposed by Kimura and Bejan [1] to observe path of heat flow. The heatline visualization technique can be employed to observe not only path of heat flow but also intensity of heat flux at any location of domain for a convection and/or conduction steady or unsteady heat/mass transfer problems. Costa [2] reviewed studies on heatline visualization technique and summarized its application. Mobedi et al. $[3,4]$ used the heatline technique to observe heat transport in the entire domain of a square cavity with thick horizontal walls. Mobedi et al. [5] also divided heatfunction equation into the diffusion and convection heatfunctions by using superposition rule. Varol et al. [6] used heatline patterns to study natural convection heat transfer of cold water near $4{ }^{\circ} \mathrm{C}$ in a thick bottom walled cavity filled with a porous medium. Kaluri et al. [7] performed a numerical study on heat distribution and thermal mixing for steady laminar natural convective flow within fluid-saturated porous square cavities by using heatline technique. Basak et al. and Kaluri et al. [8-10] performed several studies on heat transport field for various domains under different boundary conditions. Waheed [11] studied the problem of the

\footnotetext{
Communicated by W.J. Minkowycz.

* Corresponding author.

E-mail address: moghtadamobedi@lyte.edu.tr (M. Mobedi).
}

natural laminar convection in square enclosures filled with fluidsaturated porous medium by using the heatfunction formulation approach. The above literature review reveals that most of the studies on visualization of heat flow were performed on the closed spaces such as closed cavities rather than channels and ducts. Probably, the first study on observation of heat transport path in a channel was performed by Morega and Bejan [12].

Several studies on fully developed mixed convection in channels with clear fluid or completely fluid saturated porous medium were reported in literature. The energy and fluid motion equations of fully developed mixed convection flow are different than the forced convection fully developed flow since the velocity is changed with temperature in the channel. In a channel with fixed inlet mass flow, flow reversal may appear due to buoyancy effect. The buoyancy effect near the hot wall increases the fluid velocity in that region, and thus, a downward flow occurs from the open top of the channel [13]. A reversed flow situation occurs if the magnitude of the buoyancy parameter $\mathrm{Gr} / \mathrm{Re}$ exceeds a certain threshold value [14]. Parang and Keyhani [15] studied fully developed buoyancy-assisted mixed convection in a vertical annulus by using Brinkman-Extended Darcy model. Chang and Chang [16] numerically analyzed the developing mixed convection in a vertical tube partially filled with porous medium.

The aim of present study is to visualize heatline patterns in vertical plate channel with fully developed mixed convection heat transfer for three cases of a) completely filled with pure fluid, b) completely filled with fluid saturated porous medium and c) partially filled with fluid saturated porous medium. The heatfunction is determined both analytically and numerically. Analytical expressions are obtained for the 


\begin{tabular}{|c|c|}
\hline \multicolumn{2}{|c|}{ Nomenclature } \\
\hline $\mathrm{b}$ & spacing between walls (m) \\
\hline$C_{p}$ & specific heat, $\mathrm{J} \mathrm{kg}^{-1} \mathrm{~K}^{-1}$ \\
\hline $\mathrm{g}$ & acceleration due to gravity $\left(\mathrm{m} \mathrm{s}^{-2}\right)$ \\
\hline $\mathrm{Gr}_{\mathrm{c}}$ & Grashof number for the channel with clear fluid \\
\hline $\mathrm{Gr}_{\mathrm{p}}$ & Grashof number for channel with porous medium \\
\hline h & heatfunction, $\mathrm{W} \mathrm{m}^{-1}$ \\
\hline $\mathrm{H}$ & dimensionless heatfunction \\
\hline $\mathrm{J}$ & heat flux vector, $\mathrm{W} \mathrm{m}^{-2}$ \\
\hline $\mathrm{k}$ & thermal conductivity, $\mathrm{W} \mathrm{m}{ }^{-1} \mathrm{~K}^{-1}$ \\
\hline K & permeability $\left(\mathrm{m}^{2}\right)$, thermal conductivity ratio $\left(k_{f} / k_{\text {eff }}\right)$ \\
\hline M & relative viscosity, $\mu / \mu_{\text {eff }}$ \\
\hline $\mathrm{p}$ & pressure $(\mathrm{Pa})$ \\
\hline $\mathrm{P}$ & dimensionless pressure \\
\hline $\mathrm{Pe}$ & Peclet number \\
\hline $\operatorname{Re}$ & Reynolds number \\
\hline S & shape parameter, $\sqrt{M / D a}$ \\
\hline $\mathrm{T}$ & temperature $\left({ }^{\circ} \mathrm{C}\right)$ \\
\hline $\mathrm{u}, \mathrm{v}$ & axial and transverse velocity, $\left(\mathrm{m} \mathrm{s}^{-1}\right)$ \\
\hline U, V & dimensionless axial and transverse velocity \\
\hline $\mathrm{x}, \mathrm{y}$ & axial and transverse coordinate, (m) \\
\hline $\mathrm{X}, \mathrm{Y}$ & dimensionless axial and transverse coordinate \\
\hline \multicolumn{2}{|c|}{ Greek symbols } \\
\hline$\beta$ & coefficient of thermal expansion \\
\hline$\theta$ & dimensionless temperature \\
\hline$\Gamma$ & pressure gradient along channel \\
\hline$\mu$ & dynamic viscosity of the fluid $\left(\mathrm{kg} \mathrm{m}^{-1} \mathrm{~s}^{-1}\right)$ \\
\hline$\mu_{e f f}$ & effective dynamic viscosity $\left(\mathrm{kg} \mathrm{m}^{-1} \mathrm{~s}^{-1}\right)$ \\
\hline$\nu$ & kinematic viscosity of the fluid $\left(\mathrm{m}^{2} \mathrm{~s}^{-1}\right)$ \\
\hline$\rho$ & density $\left(\mathrm{kg} \mathrm{m}^{-3}\right)$ \\
\hline \multicolumn{2}{|c|}{ Subscripts } \\
\hline c & cold wall, clear fluid \\
\hline eff & effective \\
\hline f & fluid \\
\hline i & interface \\
\hline $\mathrm{p}$ & porous \\
\hline $\mathrm{h}$ & hot wall \\
\hline av & average \\
\hline ref & reference value \\
\hline
\end{tabular}

dimensionless temperature, velocity and heatfunction. For the numerical approach, a second order elliptic partial differential equation is derived and appropriate boundary conditions are defined. Then, the equation is solved numerically to determine dimensionless heatfunction in entire domain. Based on the obtained results, heatline patterns for the channels with different values of $\mathrm{Gr} / \mathrm{Re}$, Pe and Da numbers are plotted and discussed. Literature survey showed that the visualization of heat flow for fully developed mixed convection in a vertical parallel plate has not been reported in literature and the present work is an original study.

\section{The analyzed vertical parallel plate channels}

Three vertical parallel plate channels, analyzed in this study, are shown in Fig. 1. One of the channels is filled with a clear fluid (Fig. 1a), the second one completely contains fluid saturated porous medium (Fig. 1b) and the third one is partially filled with fluid saturated porous medium (Fig. 1c). The fluid flowing through the channel is assumed to be Newtonian and incompressible, while the flow is laminar, fully developed and steady. The walls of the channel are maintained at constant temperature of $T_{h}$ and $T_{c}$. The channel has a rectangular cross-section with width of $\mathrm{b}$. It is assumed that the plates are infinitely long in depth direction; fluid flows in $\mathrm{x}$ direction while $\mathrm{y}$ is perpendicular to the flow direction. The fluid properties are assumed to be constant except the density in buoyancy term of the momentum equation. Viscous dissipation and radiation heat transfer are neglected and gravity acts in $-\mathrm{x}$ direction.

\section{Mathematical formulations}

The governing equations for heat and fluid flow in the channel and their analytical solutions are presented in this section, separately.

\subsection{Channel with fully pure fluid flow}

The continuity, momentum and energy equations for a fully developed mixed convection heat transfer in a vertical channel shown in Fig. 1(a) under the Boussinesq's approximation can be written as:

$\frac{d u}{d x}=0$

$\mu_{f} \frac{d^{2} u}{d y^{2}}-\frac{d p}{d x}+\rho g \beta\left(T-T_{a v}\right)=0$

$$
\frac{d^{2} T}{d y^{2}}=0
$$

A detailed discussion on the choice of the reference fluid temperature for fully-developed mixed convection in a vertical channel was done by Barletta and Zanchini [17]. They proposed the average wall temperatures as the reference fluid temperature for fully developed mixed-convection problems in the channels. Hence, the momentum and energy equations can be non-dimensionalized as:

$\frac{d^{2} U}{d Y^{2}}+\frac{G r_{c}}{\operatorname{Re}} \theta-\Gamma=0$

$\frac{d^{2} \theta}{d Y^{2}}=0$

where Reynolds and Grashof numbers and $\Gamma$ are defined as:

$\operatorname{Re}=u_{0} b / \nu ; G r_{c}=g \beta\left(T_{h}-T_{c}\right) b^{3} / \nu^{2}, \Gamma=d P / d X$

where $u_{0}$ is inlet velocity to the channel. The following parameters are used to make the momentum and energy equations nondimensionalized:

$X=x / b, \quad Y=y / b, \quad U=u / u_{0}, \quad \theta=T-T_{a v} / T_{h}-T_{c}, \quad P=p b / u_{o} \mu_{f}$.

The dimensionless boundary conditions for momentum and energy equations (Eqs. (4) and (5)) are

On the left side; $\quad Y=0 \quad U(0)=0, \theta(0)=-0.5$

On the right side; $\quad Y=1 \quad U(1)=0 ; \theta(1)=0.5$.

The analytical solution of Eq. (5) with the boundary conditions expressed in Eqs. (8) and (9) is

$\theta=Y-0.5$ 


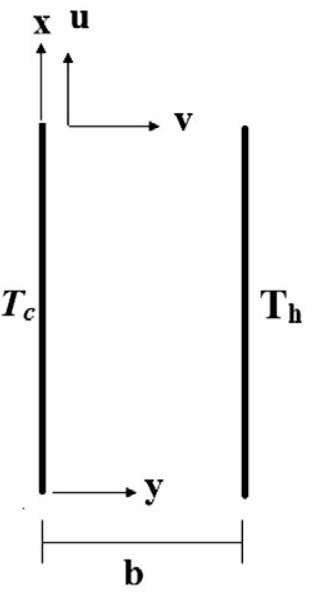

(a)

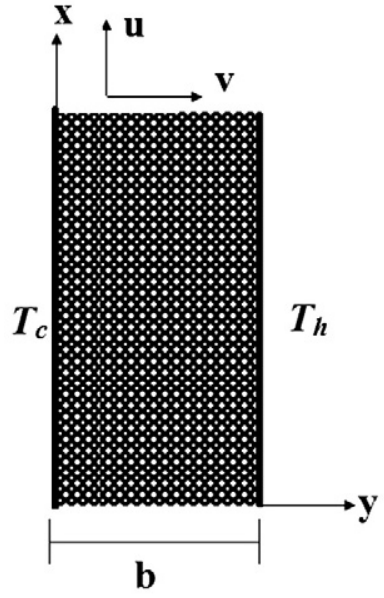

(b)

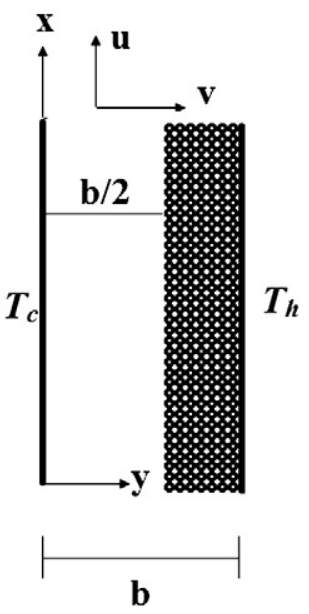

(c)

Fig. 1. Schematic view of the studied channels.

The analytical solution of motion equation (Eq. (4)) can be obtained by using the boundary conditions expressed in Eqs. (8) and (9):

$U=-\frac{G r_{c}}{\operatorname{Re}} \frac{Y^{3}}{6}+\left(\Gamma+\frac{G r_{c}}{2 \operatorname{Re}}\right) \frac{Y^{2}}{2}-\left(\frac{G r_{c}}{12 \operatorname{Re}}+\frac{\Gamma}{2}\right) Y$

where $\Gamma$ parameter still needs to be evaluated. To obtain a value for $\Gamma$, an additional equation is required. The conservation of mass in the channel can be expressed as:

$\int_{0}^{1} U(Y) d Y=1$

The integration of Eq. (11) using Eq. (12) yields $\Gamma$ value as:

$\Gamma=-12$

\subsection{Channel with saturated fluid porous medium}

The governing equations for laminar, mixed convection fully developed flow under the assumptions explained in the previous section are:

$\frac{d u}{d x}=0$

$\mu_{\text {eff }} \frac{d^{2} u}{d y^{2}}-\frac{\mu_{f}}{K} u+\rho g \beta\left(T-T_{a v}\right)-\frac{\partial p}{\partial x}=0$

$\frac{d^{2} T}{d y^{2}}=0$

As seen, Brinkman-Darcy equation is used to describe motion of fluid in the channel. Eqs. (14), (15) and (16) are needed to be solved to obtain the velocity and temperature distributions in the channel completely filled with fluid saturated porous medium. By introducing dimensionless parameters given in Eq. (7), these equations can be non-dimensionalized as follows:

$\frac{d^{2} U}{d Y^{2}}-\frac{M}{D a} U+M \frac{G r_{p}}{\operatorname{Re}} \theta-M \Gamma=0$ $\frac{d^{2} \theta}{d Y^{2}}=0$

where $G r_{p}=g \beta K\left(T_{h}-T_{c}\right) b / \nu^{2}$ which is the Darcy modified Grashof number. The dimensionless boundary conditions for motion of fluid and energy equations are identical with Eqs. (8) and (9). The analytical solution of heat transfer equation (Eq. (18)) is:

$\theta=Y-0.5$.

The analytical solution of motion equation (Eq. (17)) can be obtained by using the boundary conditions expressed in Eqs. (8) and (9):

$U=C_{1} Y+C_{2} \sinh (S(Y-1))+C_{3} \sinh (S Y)+C_{4}$

where $C_{1}, C_{2}, C_{3}$ and $C_{4}$ are constants given in Appendix $A$, and $S=\sqrt{M / D a}$. To obtain an expression for $\Gamma$, the conservation of mass can be used and therefore $\Gamma$ value is found as:

$\Gamma=\frac{\sqrt{M}}{D a\left(-\sqrt{M}+2 \sqrt{D a} \tanh \left(\frac{S}{2}\right)\right)}$.

\subsection{Channel with partially fluid saturated porous medium}

The continuity, motion and heat transfer equations, for the left half part of the channel in which clear fluid flowing is given by Eqs. (1), (2) and (3). The dimensionless forms of these equations are expressed by Eqs. (4) and (5). The dimensionless forms of motion and heat transfer equations for the right half part of the channel filled with porous medium are given by Eqs. (17) and (18). The dimensionless boundary conditions for the left half of the channel can be expressed as;

On the left side; $Y=0 \quad U(0)=0, \theta(0)=-0.5$

At interface; $Y=0.5 \quad U(0.5)=U_{i} ; \theta(0.5)=\theta_{i}$

and the dimensionless boundary conditions for porous medium layer section can be given as;

At interface; $Y=0.5 \quad U(0.5)=U_{i}, \theta(0.5)=\theta_{i}$

On the right side; $Y=1 \quad U(1)=0 ; \theta(1)=0.5$ 
where $U_{i}$ and $\theta_{i}$ are the dimensionless velocity and temperature at the interface. The analytical solutions of the heat transfer and momentum equations for the left half part with clear fluid section using the boundary conditions given by Eqs. (22) and (23) are obtained as follows;

$\theta_{c}=\left(2 \theta_{i}+1\right) Y-1 / 2$

$U_{c}=C_{5} Y+2 U_{i} Y+C_{6} Y^{2}-C_{7} Y^{3}+\Gamma\left(-\frac{Y}{4}+\frac{Y^{2}}{2}\right)$

where $C_{5}, C_{6}$ and $C_{7}$ are constants and their values are presented in Appendix A. Similarly, the temperature and velocity profile equation for fluid saturated porous region can be obtained as:

$\theta_{p}=2 \theta_{i}-Y\left(2 \theta_{i}-1\right)-1 / 2$

$U_{p}=C_{8} \sinh (S-S Y)-C_{9} \sinh (S(Y-1 / 2))-C_{10} Y+C_{11}$

where $C_{8}, C_{9}$ and $C_{10}$ and $C_{11}$ are constants and their values are given in Appendix A. $U_{p}$ and $U_{c}$ show the velocity of fluid in clear fluid and porous regions. By using condition of continuous shear stress and heat flux at the interface, $U_{i}$ and $\theta_{i}$ which are interface velocity and temperature can be found as:

$U_{i}=\frac{C_{12}(\sinh (S / 2))^{2}+C_{13} \sinh (S / 2)-C_{14}(\cosh (S)-1)}{C_{15} \sinh (S)+C_{16}(\sinh (S / 2))^{2}+C_{17} \sinh (S / 2)}$

$\theta_{i}=-\frac{K-1}{2(K+1)}$

where $\Gamma$ parameter still is needed to be evaluated and $K$ is thermal conductivity ratio $\left(K=k_{f} / k_{\text {eff }}\right)$. The conservation of mass in the channel can be expressed as:

$\int_{0}^{0.5} U_{c}(Y) d Y+\int_{0.5}^{1} U_{p}(Y) d Y=1$

The integration of Eq. (32) yields $\Gamma$ value as:

$\Gamma=\frac{C_{18}(\sinh (S / 2))^{2}+C_{19} \sinh (S / 2)+C_{20}(S / 2)-6 M S \frac{G r_{p}}{\mathrm{Re}}}{C_{21} \cosh (S / 2)+C_{22}(\sinh (S / 2))^{2}+C_{23} \sinh (S / 2)-12 M S}$

where $C_{18}, C_{19}, C_{20}, C_{21}, C_{22}$ and $C_{23}$ are constants and they are defined in Appendix $A$.

\section{Heatfunction equations}

\subsection{Mathematical definition of heatfunction}

Heatfunction and heatlines are used to visualize the path of heat flow. For a two-dimensional, incompressible and steady flow without viscous dissipation or heat generation, the heat flux vector in $\mathrm{x}$ and $\mathrm{y}$ directions can be expressed as:

$J_{x}=\rho u C_{p}\left(T-T_{r e f}\right)-k \frac{\partial T}{\partial x}$

$J_{y}=\rho u C_{p}\left(T-T_{r e f}\right)-k \frac{\partial T}{\partial y}$

where $T_{\text {ref }}$ is the reference temperature. In Cartesian coordinates, the heat flux vector can be written as:

$\vec{J}=J_{x} \overrightarrow{\mathbf{i}}+J_{y} \overrightarrow{\mathbf{j}}$
By assuming $h$ as a continuous scalar function, the heatfunction in differential form can be defined as;

$J_{x}=\rho u C_{p}\left(T-T_{r e f}\right)-k \frac{\partial T}{\partial x}=\frac{\partial h}{\partial y}$

$J_{y}=\rho v C_{p}\left(T-T_{r e f}\right)-k \frac{\partial T}{\partial y}=-\frac{\partial \mathrm{h}}{\partial x}$.

For mixed convection fully developed flow;

$v=0, \quad \frac{\partial T}{\partial x}=0$.

Therefore, for fully developed mixed convection flow, Eqs. (37) and (38) take the following form:

$j_{x}=\rho u C_{p}\left(T-T_{r e f}\right)=\frac{\partial h}{\partial y}$

$j_{y}=-k \frac{\partial T}{\partial y}=-\frac{\partial h}{\partial x}$

The Eqs. (40) and (41) can be written in the dimensionless form by using dimensionless parameters defined by Eq. (7) as;

$$
\begin{aligned}
& \frac{\partial \mathrm{H}}{\partial Y}=\operatorname{Pe} U(\theta+1 / 2) \\
& \frac{\partial H}{\partial X}=\frac{\partial \theta}{\partial Y}
\end{aligned}
$$

where $T_{\text {ref }}$ is accepted as $T_{c}$, and $\mathrm{H}$ and Pe are dimensionless heatfunction and Peclet number:

$$
\begin{aligned}
& P e=\frac{\rho c_{p} u_{0} b}{k} \\
& \mathrm{H}=\frac{h}{k\left(T_{\mathrm{h}}-T_{c}\right)} .
\end{aligned}
$$

\subsection{Analytical expression for heatfunction in the channel with complete- ly clear fluid}

Thermal conductivity plays an important role on definition of dimensionless heatfunction. There is no doubt that for the channel with completely clear fluid, $k$ is the thermal conductivity of fluid $\left(k_{f}\right)$ and consequently $\mathrm{H}_{f}=\mathrm{h} /\left(k_{f}\left(T_{\mathrm{h}}-T_{c}\right)\right)$ andPe $\mathrm{P}_{f}=\rho c_{p} u b / k_{f} . \mathrm{H}_{f}$ and $\mathrm{Pe}_{f}$ are dimensionless heatfunction and Peclet number for the clear fluid channel. Another important point in determination of heatfunction is the reference value for heatfunction. In this study, the value of heatfunction at the origin is assumed zero, $\mathrm{H}_{f}(0,0)=0$. The following equations can be written based on taking integral from Eqs. (42) and (43):

$$
\begin{aligned}
& \mathrm{H}_{f}(X, Y)=\int \mathrm{Pe} U(\theta+1 / 2) d Y+C_{24}(X) \\
& \mathrm{H}_{f}(X, Y)=X+C_{25}(Y) .
\end{aligned}
$$

The heatfunction should be valid for the whole of domain. The value of $C_{25}(0)$ can be found by applying Eq. (47) for the origin:

$\mathrm{H}_{f}(0,0)=\mathrm{C}_{25}(0)=0$

Eqs. (46) and (47) are also valid for $\mathrm{Y}=0$ surface, then

$\mathrm{H}_{f}(X, 0)=C_{24}(X)+C_{26}$ 
$\mathrm{H}_{f}(X, 0)=X+C_{25}(0)$

From the above equations, $C_{24}(X)$ can be found as:

$C_{24}(X)=X+C_{26}$

Therefore, the heatfunction can be expressed as:

$\mathrm{H}_{f}(X, Y)=\int \mathrm{Pe} U(\theta+1 / 2) d Y+X+C_{26}$.

The heatfunction equation for channel with fully clear fluid can be obtained as:

$\mathrm{H}_{\mathrm{f}}(\mathrm{X}, \mathrm{Y})=\mathrm{X}+\operatorname{Pe}\left(-\frac{\mathrm{Gr}_{\mathrm{c}}}{\operatorname{Re}} \frac{\mathrm{Y}^{5}}{30}+\left(\frac{\Gamma}{8}+\frac{\mathrm{Gr}_{\mathrm{c}}}{16 \operatorname{Re}}\right) \mathrm{Y}^{4}+\left(-\frac{\Gamma}{6}-\frac{\mathrm{Gr}_{\mathrm{c}}}{36 \operatorname{Re}}\right) \mathrm{Y}^{3}\right)$

The value of $C_{26}$ and integral constant are zero since $\mathrm{H}(0,0)=0$.

\subsection{Analytical expression for heatfunction in channel with completely} porous medium

Eqs. (42) and (43) are valid for dimensionless heatfunction in a channel with completely porous medium. However, the effective thermal conductivity of porous medium should be used for the definition of dimensionless heatfunction and Peclet numbers and consequently, $\mathrm{H}_{p}=\mathrm{h} /\left(k_{\text {eff }}\left(T_{\mathrm{h}}-T_{c}\right)\right)$ and $P e_{p}=\rho c_{p} u b / k_{\text {eff. }}$ Similar procedure to obtain Eq. (53) can be performed, and the heatfunction equation for the channel filled with fluid saturated porous medium can be obtained as:

$$
\begin{aligned}
\mathrm{H}_{\mathrm{P}}(\mathrm{X}, \mathrm{Y})= & \mathrm{X}+\mathrm{Pe}\left(\frac{\mathrm{C}_{1}}{3} \mathrm{Y}^{3}-\frac{1}{\mathrm{~S}^{2}}\left(\mathrm{C}_{3} \sinh (\mathrm{SY})-\mathrm{S}\left(\mathrm{C}_{2} \mathrm{Y} \cosh (\mathrm{S}(\mathrm{Y}-1))\right.\right.\right. \\
& \left.\left.\left.+\mathrm{C}_{3} \mathrm{Y} \cosh (\mathrm{SY})\right)+\mathrm{C}_{2} \sinh (\mathrm{S}(\mathrm{Y}-1))\right)+\mathrm{C}_{4} \frac{\mathrm{Y}^{2}}{2}+\mathrm{C}_{2} \sinh (\mathrm{S})\right)
\end{aligned}
$$

where $C_{1}, C_{2}, C_{3}$ and $C_{4}$ are constants and the related equations are given in Appendix A.

4.4. Analytical expression for heatfunction in channel with partially filled porous medium

The dimensionless heatfunction for the clear fluid region can be found from the following equation:

$$
\begin{aligned}
& \frac{\partial \mathrm{H}_{f}}{\partial Y}=P e_{f} U(\theta+1 / 2) \\
& \frac{\partial \mathrm{H}_{f}}{\partial X}=\frac{\partial \theta}{\partial Y}=\left(2 \theta_{i}+1\right) .
\end{aligned}
$$

Heatfunction differential equation for the porous medium region can also be found from the following equations:

$$
\begin{aligned}
& \frac{\partial \mathrm{H}_{p}}{\partial Y}=P e_{p} U(\theta+1 / 2) \\
& \frac{\partial \mathrm{H}_{p}}{\partial X}=\frac{\partial \theta}{\partial Y}=\left(1-2 \theta_{i}\right) .
\end{aligned}
$$

The comparison between the definitions of dimensionless heatfunction for clear fluid and porous medium filled regions (i.e., $\mathrm{H}_{f}=$ $\mathrm{h} /\left(k_{f}\left(T_{\mathrm{h}}-T_{c}\right)\right)$ and $\left.\mathrm{H}_{p}=\mathrm{h} /\left(k_{\text {eff }}\left(T_{\mathrm{h}}-T_{c}\right)\right)\right)$ shows that the definitions of $\mathrm{H}_{f}$ and $\mathrm{H}_{p}$ are not the same on the solid-fluid interface and a discontinuity exits. A point on the interface has two dimensionless heatfunction values due to different definitions of $\mathrm{H}_{f}$ and $\mathrm{H}_{p}$. Finding a relation between $\mathrm{H}_{f}$ and $\mathrm{H}_{p}$ may be a solution for this difficulty.

$\mathrm{H}_{p}=\frac{\mathrm{h}}{k_{f}\left(T_{\mathrm{h}}-T_{c}\right)} \frac{1}{K}=\mathrm{H}_{f} K$

By substituting Eq. (59) into Eqs. (57) and (58), the following equations can be obtained for the porous medium region in the channel.

$\frac{\partial \mathrm{H}_{f}}{\partial Y}=P e_{f} U(\theta+1 / 2)$

$\frac{\partial \mathrm{H}_{f}}{\partial X}=\frac{1}{K} \frac{\partial \theta}{\partial Y}=\frac{1}{K}\left(1-2 \theta_{i}\right)$

As seen, Eqs. (60) and (61), the heatfunction for porous region is defined based on the fluid thermal conductivity. By the other words, the fluid thermal conductivity is used to define dimensionless heatfunction in the entire channel, and no discontinuity of dimensionless heatfunction is faced at the interface. Similar method, used to find Eq. (53), can be employed to determine dimensionless heatfunction for the clear and porous medium regions. Dimensionless heatfunction for the left half of the channel can be obtained as:

$\begin{aligned} H_{f}= & \left(2 \theta_{i}+1\right) X \\ & +\frac{\left(\left(2 \theta_{i}+1\right) \mathrm{PeY}^{3}\left(40 C_{5}-10 \Gamma+80 U_{i}+30 C_{6} Y+15 \Gamma Y-24 C_{7} Y^{2}\right)\right.}{120} .\end{aligned}$

The dimensionless heatfunction for right half of the channel region is:

$$
\begin{aligned}
\mathrm{H}_{f}= & \left(1-2 \theta_{i}\right) X / K+C_{11} 2 \theta_{i} P e Y-C_{27} Y^{2}+C_{28} Y^{3}-C_{29} \sinh (S(1-Y)) \\
& +C_{30} \sinh (S(Y-1 / 2))-C_{31} \cosh (S(1-Y))-C_{32} \cosh (S(Y-1 / 2)) \\
& -C_{33} Y \cosh (S(1-Y))-C_{34} Y \cosh (S(Y-1 / 2))+C_{35}-C_{36} .
\end{aligned}
$$

\subsection{Elliptic PDE for heatfunction}

In this section, an elliptic partial differential equation for dimensionless heatfunction is derived. Taking derivatives with respect to $\mathrm{y}$ and $\mathrm{x}$ from Eqs. (40) and (41) leads to:

$\rho C p \frac{\partial}{\partial y}\left(u\left(T-T_{r e f}\right)\right)=\frac{\partial^{2} h}{\partial y^{2}}$

$-k \frac{\partial^{2} T}{\partial x \partial y}=-\frac{\partial^{2} h}{\partial x^{2}}$

For mixed convection fully developed flow, $\frac{\partial}{\partial y}\left(\frac{\partial T}{\partial x}\right)=0$. The summation of Eqs. (64) and (65) yields partial differential heatfunction equation:

$\frac{\partial^{2} \mathrm{~h}}{\partial x^{2}}+\frac{\partial^{2} \mathrm{~h}}{\partial y^{2}}=\rho C p \frac{\partial}{\partial y}\left(u\left(T-T_{r e f}\right)\right)$

The above heatfunction equation, which is an elliptical partial differential equation, can be written in dimensionless form. 
$\frac{\partial^{2} \mathrm{H}}{\partial X^{2}}+\frac{\partial^{2} \mathrm{H}}{\partial Y^{2}}=P e \frac{\partial}{\partial y}\left(U\left(\theta+\frac{1}{2}\right)\right)$

where $T_{\text {ref }}$ is accepted as $T_{c}$. The solution of Eq. (67) yields the distribution of heatfunction in the channel. The boundary conditions for heatfunction equation can be written by using Eqs. (42) and (43).

For $Y=0 \mathrm{H}(X, 0)=\left.\int_{X=0}^{X} \frac{\partial \theta}{\partial Y}\right|_{Y=0} d X$

For $Y=1 \mathrm{H}(X, 1)=\left.\int_{X=0}^{X} \frac{\partial \theta}{\partial Y}\right|_{Y=1} d X$

For $X=0 \mathrm{H}(0, Y)=\int_{Y=0}^{Y} P e U(\theta+1 / 2) d Y$

For $X=X_{0} \mathrm{H}\left(X_{0}, Y\right)=\int_{Y=0}^{Y} \operatorname{PeU}(\theta+1 / 2) d Y$

where $X_{0}$ is the ratio of $\mathrm{b} / \mathrm{L}$. The above differential equation is valid for completely clear fluid channel or completely porous medium filled channel. For the channel partially filled with porous medium, $\theta, \mathrm{H}$ and $P e$ are replaced with $\theta_{c} \mathrm{H}_{f}, P e_{f}$ for the left half of the channel, and the same parameters are replaced with $\theta_{p}, \mathrm{H}_{p}$ and $P e_{p}$ for the right half of the channel. Then, $\mathrm{KH}_{f}$ can be written instead of $\mathrm{H}_{p}$. The value of $\mathrm{H}_{f}$ at the interface can be calculated by the following relation:

For $Y=0.5 \quad H(X, 0.5)=\left.\int_{X=0}^{X} \frac{\partial \theta_{f}}{\partial Y}\right|_{Y=0.5} d X$

4.6. Relation between dimensionless heat transfer rate and dimensionless heatfunction

The heat received by cold wall (left wall) of a channel with unit height can be calculated by the following relation:

$\left.\mathrm{j}\right|_{y=0}=\frac{\left(T_{h}-T_{c}\right)}{\mathrm{R}}=\int_{x=0}^{x=l} \mathrm{~h}(x, 0) \mathrm{dx}$

where $\mathrm{j}$ is heat flux to the left wall and $\mathrm{R}$ is thermal resistance which is $b / k$. By substituting dimensionless parameters defined by Eq. (7), the dimensionless heat transfer rate from right to the left wall for a channel with dimensionless height of 2 will be obtained as:

$\left.j\right|_{Y=0}=\mathrm{H}(2,0)=2$.

Hence, for both channels with completely clear fluid and completely porous medium, the maximum value of dimensionless heatfunction at the left wall should be 2 . For partially porous medium filled channel, the situation is different. Heat transfer rate received by the left wall depends on thermal conductivity ratio and porous layer thickness and it can be calculated from the following relation:

$\left.\mathrm{j}\right|_{y=0}=\frac{\left(T_{\mathrm{h}}-T_{c}\right)}{\sum \mathrm{R}}=\int_{x=0}^{x=l} \mathrm{~h}(x, 0) d x$ where $\sum R$ is thermal resistance between the hot and cold walls and it can be defined as:

$\sum \mathrm{R}=\frac{l_{p}}{k_{p}}+\frac{l_{f}}{k_{f}}$

where $k_{p}$ and $k_{f}$ are effective thermal conductivity of the porous and fluid thermal conductivity, $l_{p}$ and $l_{f}$ are the thickness of porous layer and section with pure fluid flow. Since the dimensionless heatfunction is defined according to the fluid thermal conductivity, the dimensionless heatfunction at the left wall can be determined by following relation:

$\left.\mathrm{j}\right|_{Y=0}=\frac{2}{\sum R^{\prime}}=\mathrm{H}_{f}(2,0)$

where $R^{\prime}$ is normalized thermal resistance according to the fluid thermal conductivity. For the present problem, the value of $R^{\prime}$ can be calculated from the following relation:

$\sum R^{\prime}=0.5 K+0.5$

\section{Result and discussion}

Both numerical and analytical results (the velocity profile, temperature and heatfunction distribution) were found and compared for all presented results. The numerical and analytical results are identical when $101 \times 101$ number of nodes is used in the numerical approach. Furthermore, the velocity profiles are also compared with the profiles reported in the literature. For instance, the velocity profiles of the present work and the profiles reported by Aung and Worku [14] for the channels with clear fluid and by Degan and Vasseur [18] for the channels filled with fluid saturated porous medium are compared and good agreement between the results is observed.

\subsection{Heatline patterns in the channel with pure fluid flow}

Fig. 2 shows the velocity profile, temperature distribution and heatline patterns in a channel with two values of $G r_{c} / \operatorname{Re}$ of 1 and 400 , and three Peclet numbers of $0.01,1$ and 5 . The velocity profile and temperature distribution are shown in the first column while the heatline patterns are in the second, the third and fourth columns for three Peclet numbers of $0.01,1$ and 5, respectively. The velocity profile, temperature distribution and heatline patterns for the channel with $G r_{c} / \operatorname{Re}=1$ are shown in the first column of Fig. 2(a). Forced convection is dominant and velocity profile is almost parabolic and a linear temperature variation exits in the channel. The heatline patterns in the channel with Peclet number of 0.01 are not affected from fluid flow and heat path is almost horizontal. By increasing Peclet number from 0.01 to 1 , the heatline patterns are considerably changed and the convection heat transfer becomes stronger. For $\mathrm{Pe}=1$, the heatlines are not horizontal and the effect of vertical convection transport can be observed. The convection heat transport in vertical direction is improved for the channel with $\mathrm{Pe}=5$. Heat takes a long distance in vertical direction to be transferred from the right to left wall. It should be mentioned that the maximum heatfunction value at the left wall, showing the dimensionless heat transfer from the right to left wall, is $\mathrm{H}_{\max }=2$ for three Peclet numbers, as expected. Fig. 2(b) shows the velocity profile, temperature distribution, and heatline patterns in a channel with $G r_{c} / \operatorname{Re}=400$ for the same Peclet numbers of Fig. 2(a). The increase of $G r_{c} /$ Re ratio, increases mass flow in upward direction and that is why a reverse flow occurs in the channel. The comparison of Fig. 2(a) and (b) shows that the change of velocity profile in the channel does not affect heatline distribution in the channel for $\mathrm{Pe}=0.01$ since the conduction heat transfer from the right to left wall is the dominant mode of heat transport between two plates and heat moves horizontally from the right to left wall. For 

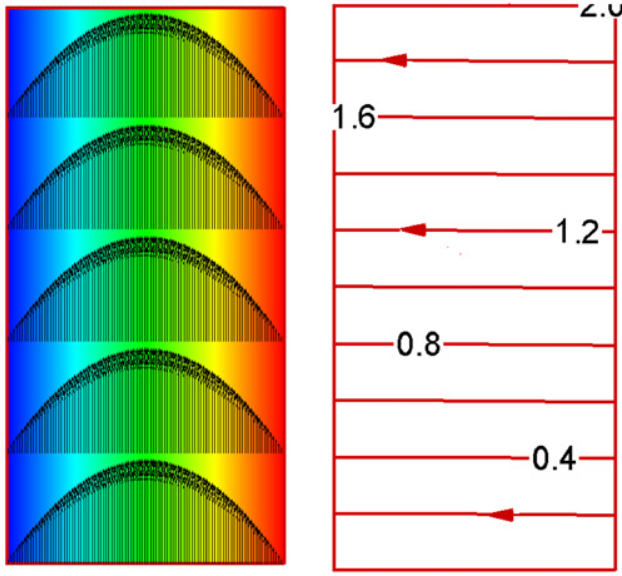

$\mathrm{Pe}=0.01$
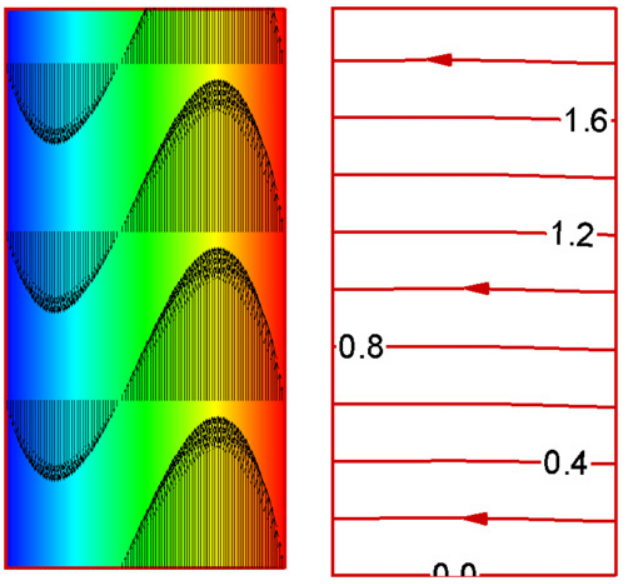

$\mathrm{Pe}=0.01$

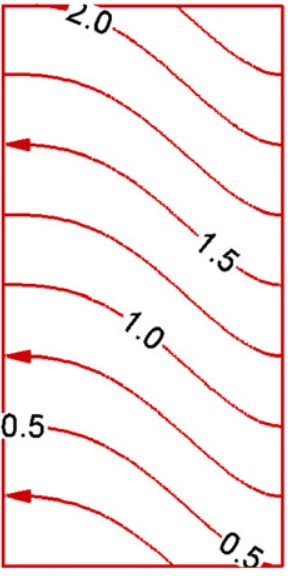

$\mathrm{Pe}=1$

(a)

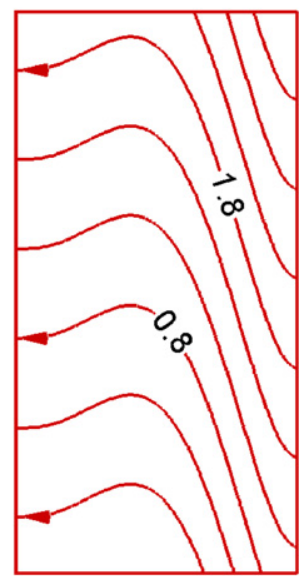

$\mathrm{Pe}=1$

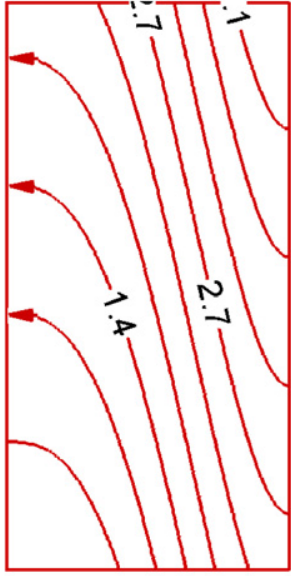

$\mathrm{Pe}=5$

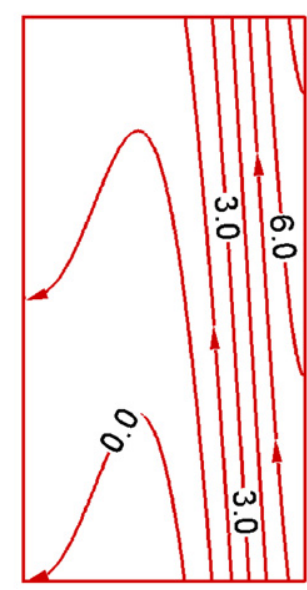

$\mathrm{Pe}=5$

(b)

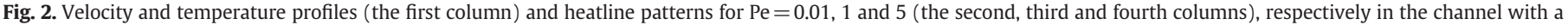
clear fluid, a) $\mathrm{Gr}_{\mathrm{c}} / \mathrm{Re}=1$, b) $\mathrm{Gr}_{\mathrm{c}} / \mathrm{Re}=400$.

$P e=1$, the effects of the convection mode of heat transport and the effect of reverse flows on heatline patterns can be observed. Heat is separated from the right wall and goes in upward direction due to the strong convection. After a distance, the direction of heat transport is changed and heat flows in downward direction due to the reverse flow and finally it is received by the left wall. Further distortion of heatline patterns can be observed in the channel with $\mathrm{Pe}=5$. In the channels with high value of Peclet number (i.e., Pe $=5$ ), for which convective heat transport is dominant, heatline patterns are highly affected from fluid flow. It should be mentioned that for the clear fluid channel with $G r_{c} / \operatorname{Re}=400$, the dimensionless heat transfer rate is 2 since a linear temperature distribution across the channel exists.

\subsection{Heatline patterns in the channel filled with fluid saturated porous media}

Fig. 3 shows the velocity profile, temperature distribution and heatline patterns in a porous medium filled channel with $D a=10^{-4}$ and two values of $G r_{p} / \operatorname{Re}$ as 0.01 and 5. It shows the heatline patterns for three Peclet numbers of $0.01,1$ and 5. Similar to Fig. 2(a), for low values of $G r_{p} / \operatorname{Re}=0.01$, forced convection is dominant and a symmetrical velocity profile is observed in the channel. For the low values of Peclet number (i.e. $\mathrm{Pe}=0.01$ ), the conduction mode of heat transfer from the right to left wall is dominant and heat flows horizontally. By increasing Peclet number from 0.01 to 1.0, the convection in vertical direction affects heatline patterns and heat separated from the right wall takes a vertical distance to be received by the left wall. The effect of vertical flow on heatline patterns is clearly observed in the channel with $\mathrm{Pe}=5$ in which a strong convective transport exists. Fig. 3(b) shows that by increasing $\mathrm{Gr}_{p} /$ Re from 0.01 to 5 , a reverse flow occurs in the channel. This reversal flow does not affect heatline patterns for the channel with $\mathrm{Pe}=0.01$ due to strong heat conduction from the right to left wall. However, the heatline distribution in the channel with $\mathrm{Pe}=5$ is highly influenced from the reverse flow due to the encountering effect of convection heat transport in vertical direction.

Fig. 4 compares the velocity and heatline patterns in the channel with clear fluid $\left(G r_{c} / \operatorname{Re}=1\right)$ and the channel completely filled with saturated fluid porous medium $\left(G r_{p} / \operatorname{Re}=0.01, D a=10^{-4}\right)$ when $\mathrm{Pe}=5$. The velocity profile of the channel with completely porous medium is flattened due to the obstacles in the porous medium. The 

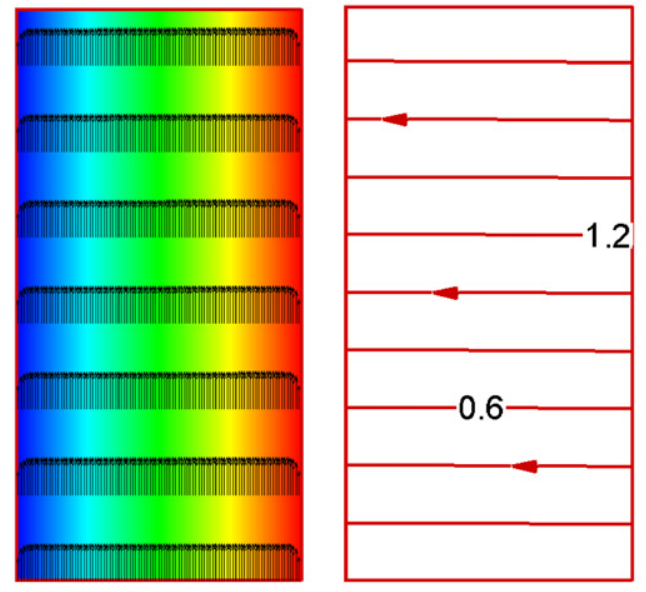

$\mathrm{Pe}=0.01$
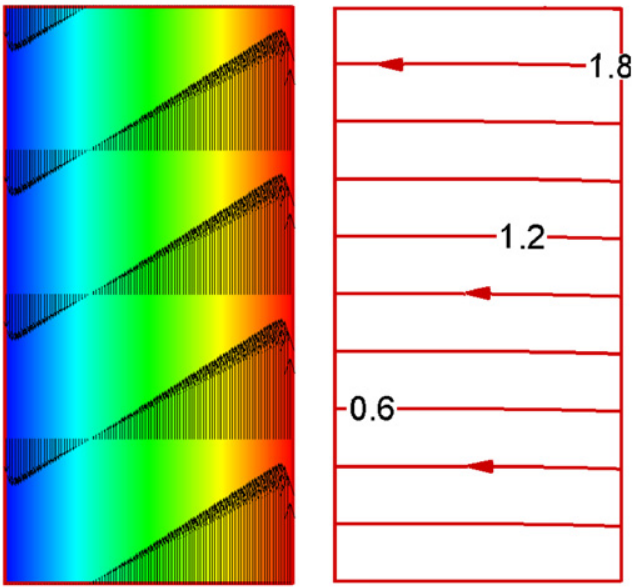

$\mathrm{Pe}=0.01$

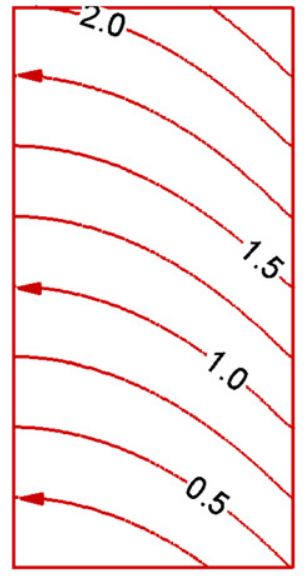

$\mathrm{Pe}=1$

(a)

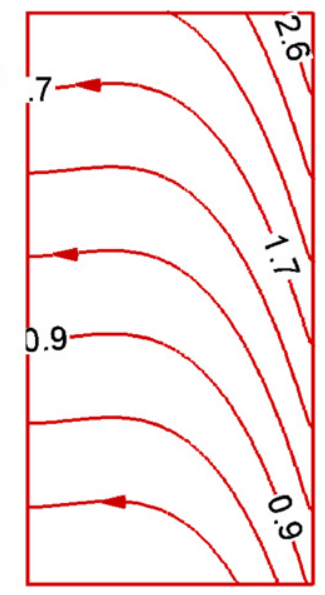

$\mathrm{Pe}=1$

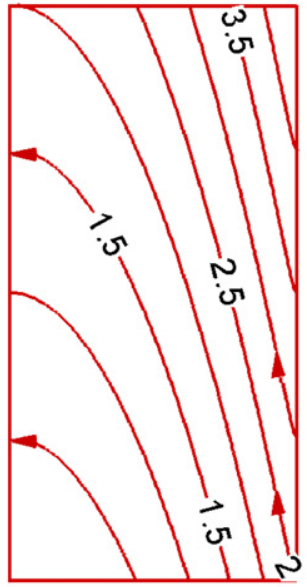

$\mathrm{Pe}=5$

(b)

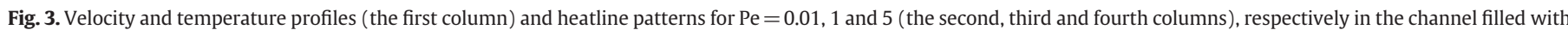
saturated porous medium, a) $\mathrm{Gr}_{\mathrm{p}} / \mathrm{Re}=0.01$, b) $\mathrm{Gr}_{\mathrm{p}} / \mathrm{Re}=5$.

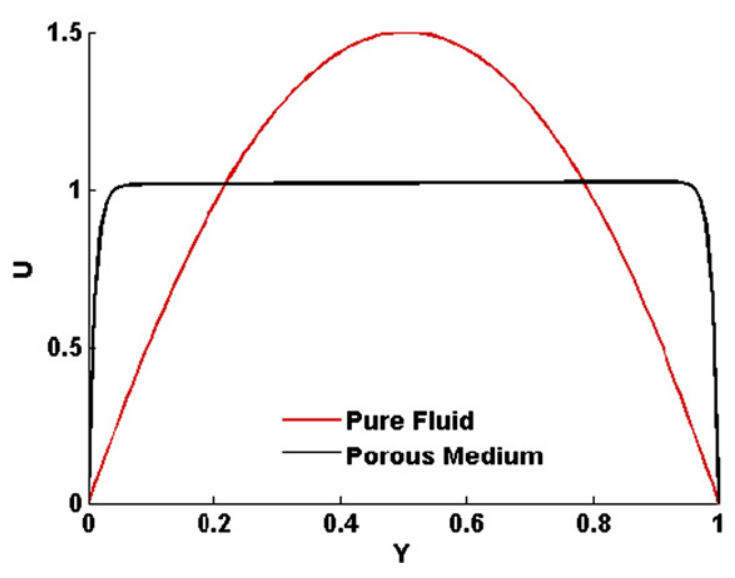

(a)

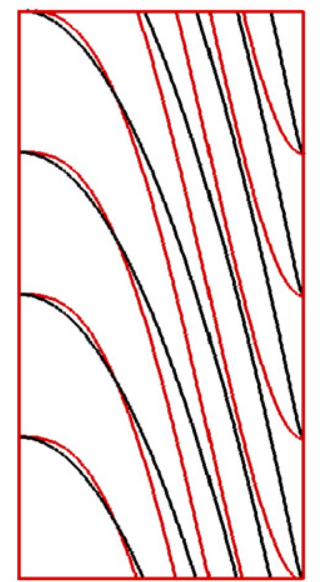

(b)

Fig. 4. Comparison of heatline for clear fluid $\left(\mathrm{Gr}_{\mathrm{c}} / \mathrm{Re}=1\right)$ and completely porous medium channel $\left(\mathrm{Gr}_{\mathrm{p}} / \mathrm{Re}=0.01, \mathrm{Da}=10^{-4}\right)$ when $\mathrm{Pe}=5$. 
temperature variation for both channels is identical, and temperature linearly changes from right to the left wall. Fig. 4(b) compares the heatline patterns of the both channels. As seen, there is a difference in heatline patterns due to small changes in the velocity profiles. This figure shows that for high values of Peclet number, how the small changes in velocity profile can affect heatline patterns.

\subsection{Heatline patterns in the channel partially filled with porous medium}

Fig. 5 shows the velocity profile, temperature distribution and heatline patterns for channels partially filled with porous medium. The thickness of the porous layer is 0.5 while the channel thickness is 1. The right half of the channel is filled with porous medium while the pure fluid flows in the left half of the channel. Fig. 5(a) shows the velocity profile, temperature distribution and heatline patterns for the channel with $G r_{c} / \operatorname{Re}=1, D a=10^{-4}$ and $K=0.05$ which refers to high effective thermal conductivity of porous medium compared to that of clear fluid. As seen from this figure, the left half of the channel, filled with high conductive porous medium, is hot $(\theta=0.5)$ and temperature is almost uniform. The dimensionless temperature linearly changes from 0.5 to -0.5 in the right half of the channel. The value $G r_{c} / \operatorname{Re}=1$ refers to the strong forced convection heat transfer in the channel. The value of Darcy is low (i.e. $\mathrm{Da}=10^{-4}$ ) and a parabolic velocity profile occurs in the left half of the channel where the fluid can flow easily. The fluid flow in the right half of the channel is weak compared to left side due to the low value of Darcy number. For Pe $=0.01$, the conduction mode of heat transfer from the right to left wall is dominant and that is why heat flows horizontally from the hot to cold wall. By increasing Peclet number from 0.01 to 1 , the direction of heat flow in the right half region is not changed. The convection strength in vertical direction is weak while the conduction heat transport from the right wall to the interface is strong. That is why, heat flows horizontally in the right half of the channel. In the left half of the channel, the effect of convection heat transfer in vertical direction is considerably higher than the conduction heat transfer in horizontal direction. The path of heat transfer is changed in the left region of the channel. Heat flows upward due to the strong convection effect while it flows toward the cold wall and finally it is received by the right wall. By increasing Peclet number from 1 to 5 , the heat path does not change in the left half of the channel due to the strong conduction mode of heat transfer from the right wall toward the
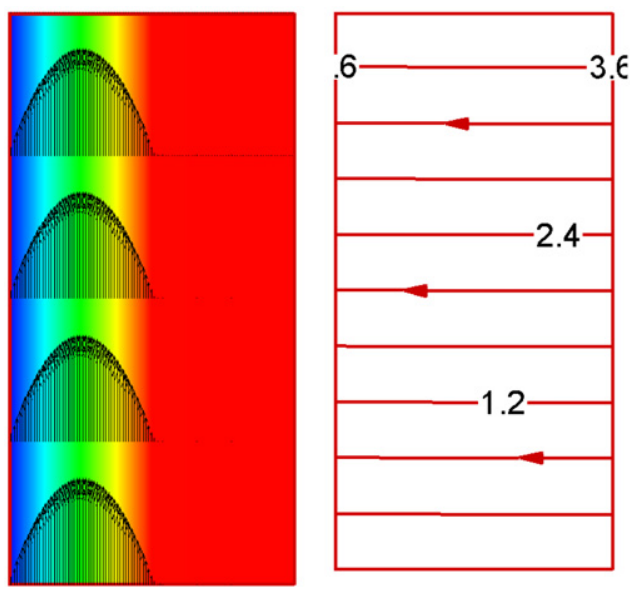

$\mathrm{Pe}=0.01$

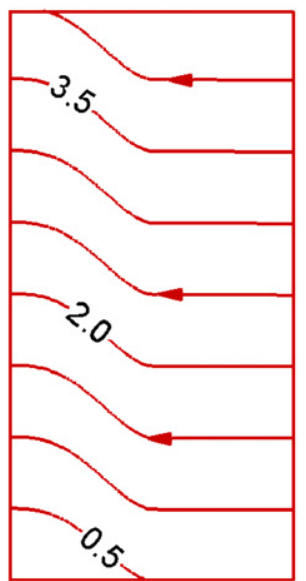

$\mathrm{Pe}=1$
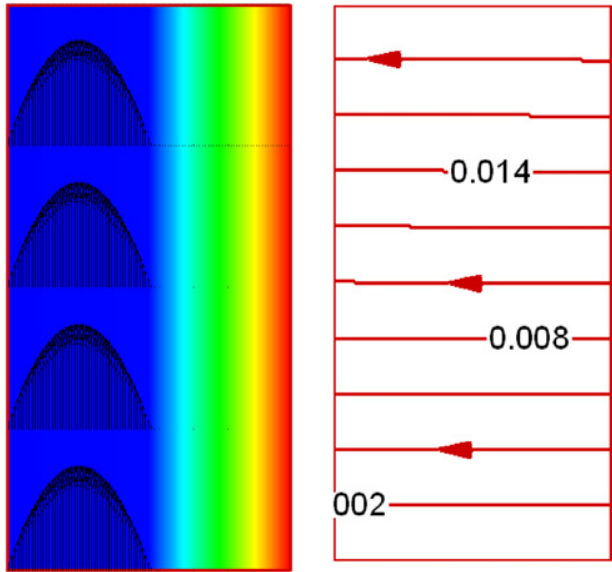

$\mathrm{Pe}=0.01$ (a)

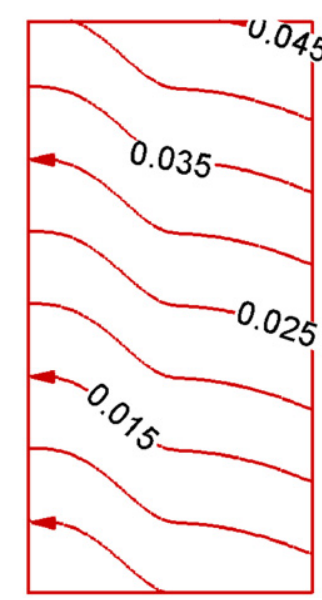

$\mathrm{Pe}=1$

(b)

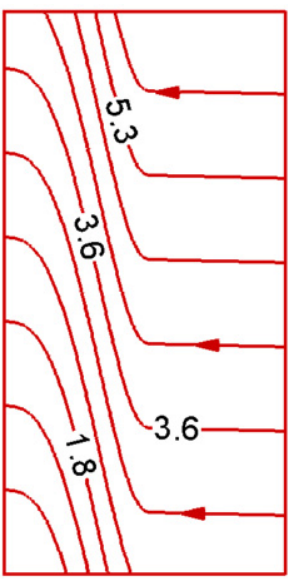

$\mathrm{Pe}=5$

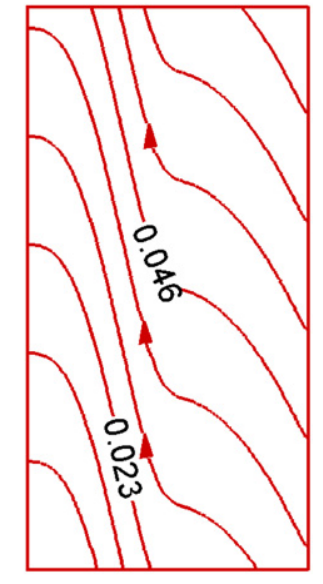

$\mathrm{Pe}=5$

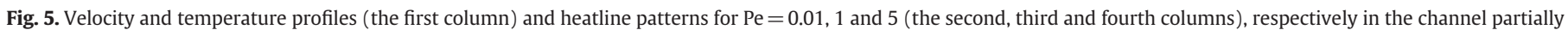
filled with saturated porous medium, $\mathrm{Gr}_{\mathrm{c}} / \mathrm{Re}=1, \mathrm{Da}=10^{-4}$ a) $\mathrm{K}=0.05$, b) $\mathrm{K}=100$. 
interface. However, in the left half region, heat takes a longer distance in vertical direction due to the strong convection heat transfer in vertical direction. The maximum value of dimensionless heatfunction at the left wall is 3.81 indicating that by inserting of high conductive porous layer heat transfer rate from the right to left increases.

The velocity profile, temperature distribution and heat flow patterns in the channel with $G r_{c} / \operatorname{Re}=1, D a=10^{-4}$ and $K=100$ are shown in Fig. 5(b). The heatline patterns are plotted for three different Peclet numbers of $0.01,1$ and 5 . As seen from the first column, a linear temperature distribution is observed in the right half of the channel while temperature is uniform in the left half (i.e., $\theta=-0.5$ ) due to the high thermal conductivity of pure fluid compared to the effective thermal conductivity of porous medium region. The velocity is small in the right half of the channel while a parabolic velocity profile is observed in the left half. For low Peclet number of 0.01, the heat flow is horizontal from the hot to cold wall. For $\mathrm{Pe}=1$, in the right half of the channel, the conduction heat transport in horizontal direction from the right wall to interface is weak and it is comparable with convective heat transport in the vertical direction. That is why; heat separated from the right wall moves vertically due to the convective heat transport and then it moves toward the interface. In the left half of the channel, the convection heat transport in vertical direction (i.e., thermal energy transported in vertical direction) is comparable with the conduction heat transport in horizontal direction since the fluid thermal conductivity is high. Hence, heat moves vertically when it moves toward the left wall. By increasing Peclet number from 1 to 5 , the same heat transport paths are valid. However, the heatlines become more vertical due to the stronger convection heat transport in vertical direction. The maximum dimensionless heatfunction at the left wall that is 0.04 showing insertion of porous layer with low thermal conductivity reduces heat transfer between the walls.

Fig. 6(a) shows the velocity profile, temperature distribution and heatline patterns in the channel with $G r_{c} / \operatorname{Re}=2000$ and $D a=10^{-4}$ when $K=0.05$. Similar to Fig. 5 , the heatline patterns are plotted for Peclet numbers of $0.01,1$ and 5 . As seen from the first column of Fig. 6(a), the temperature in the right half of the channel is uniform as $\theta=0.5$ due to high effective thermal conductivity of porous medium. Consequently, the interface temperature is $\theta=0.5$ and a linear temperature change is observed in the left half of the channel. The velocity is small in the left region due to low value of Darcy number and a reverse flow occurs in the left half of the channel due to high buoyancy effect. The second column of Fig. 6(a) shows heatline patterns in
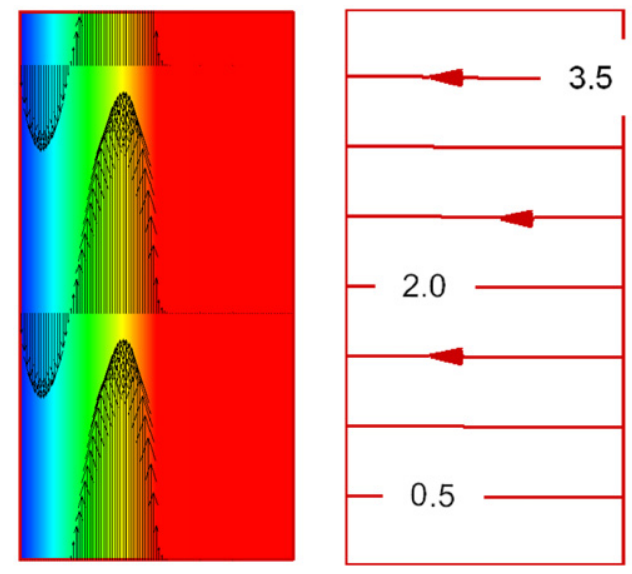

$\mathrm{Pe}=0.01$

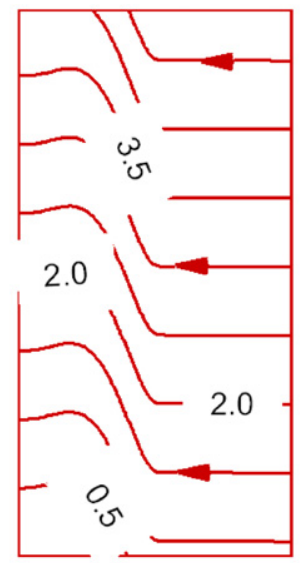

$\mathrm{Pe}=1$

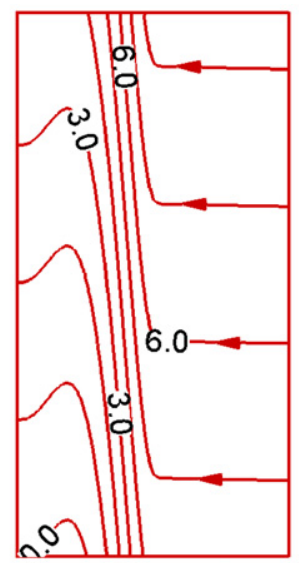

$\mathrm{Pe}=5$

(a)

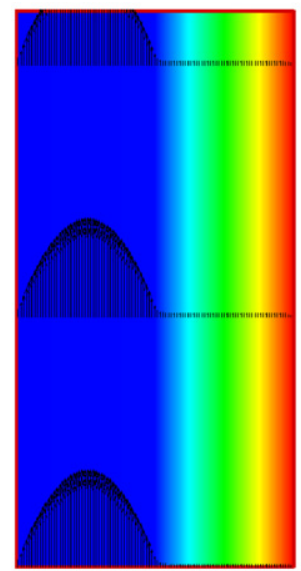

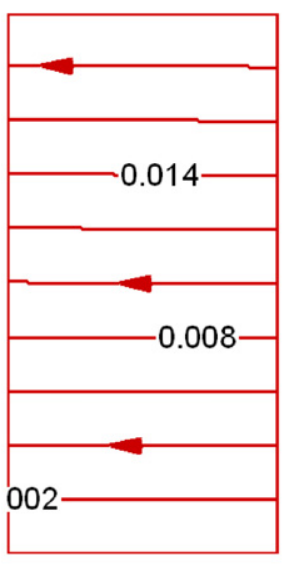

$\mathrm{Pe}=0.01$

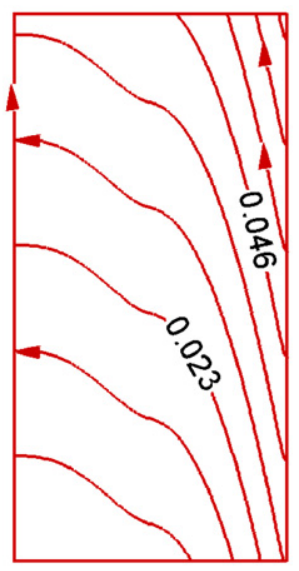

$\mathrm{Pe}=1$

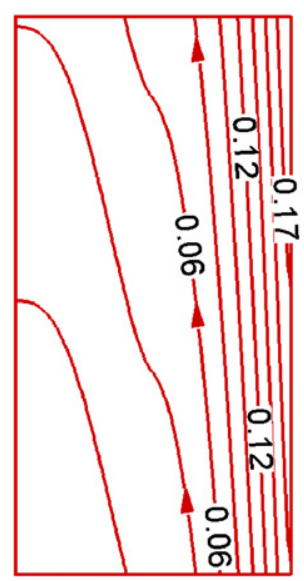

$\mathrm{Pe}=5$

(b)

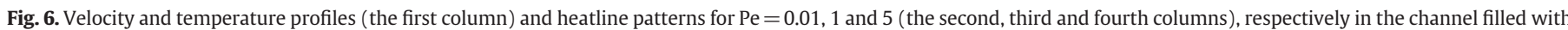
saturated porous medium, $\mathrm{Gr}_{\mathrm{c}} / \mathrm{Re}=2000, \mathrm{Da}=10^{-4}$, a) $\left.\mathrm{K}=0.05 \mathrm{~b}\right) \mathrm{K}=100$. 
the channel when $P e=0.01$. As expected, heat is transported horizontally from the hot to the cold wall for $\mathrm{Pe}=0.01$. For $\mathrm{Pe}=1$, heat transfers horizontally in the right half and it moves upward in the left half of the channel due to the strong convective heat transport. Then, it attains a maximum point. After this point, heat moves downward due to the reverse flow that occurs in the region close to the cold wall. By increasing Peclet number from 1 to 5 , the effect of convection heat transfer in vertical direction increases and heat moves a long distance in the vertical direction in the left half of the channel.

The parameters of Fig. 6(b) are the same with the parameters of Fig. 6(a), except thermal conductivity ratio which is 0.01 refers to low thermal conductivity of the right half compared to the left one. As seen from the first column of Fig. 6(b), the temperature linearly changes in the right half of the channel and it is uniform in the left half as $\theta=-0.5$. No buoyancy effect exists in the left half of the channel since both the left wall and interface are at $\theta=-0.5$. A parabolic velocity profile occurs in this region due to the fixed inlet mass flow rate. For $\mathrm{Pe}=0.01$, as expected, the heat transport is horizontal. For $\mathrm{Pe}=1$, in the right region, the convection heat transport in vertical direction is higher than conduction heat transport in horizontal direction. It should be reminded that in the right half of the channel, the conduction heat transfer from the right wall to the interface is weak due to the low effective thermal conductivity of porous medium. That is why; heat moves a distance in vertical direction when it goes toward the interface. After passing of heat from the interface, in the left half of the channel, heat moves more horizontally due to high conduction heat transfer form the interface to the left wall. The same comments are valid for the channel with $P e=5$, however the effect of upward flow on heatline patterns increases as seen from the fourth column of Fig. 6(b).

\section{Conclusion}

Analytical and numerical studies are performed to observe heatline patterns in a vertical channel with mixed convection. Analytical expressions for determination of velocity, temperature and heatfunction are presented for three channels of a) filled with clear fluid, b) filled with fluid saturated porous medium and c) partially filled with fluid saturated porous medium. It is observed that the use of heatfunction concept can explain the competition between the horizontal conduction heat transport and vertical convection mode of heat transfer in the channel. Based on the obtained results the following remarks can be concluded:

- The path of heat in the channel is highly affected from Peclet number. For low Peclet number, heat transfer is horizontal; however heat takes a distance in vertical direction for high Peclet number.

- For the channel with high Peclet number, heatline patterns are highly influenced from the velocity profile since convection heat transfer is strong. If a reverse flow occurs in the channel, the heat direction is also changed and heat flows downward.

- For the channel with partially porous medium, comments on the heatline patterns become more difficult since the thermal conductivity ratio should also be taken into account. When the effective thermal conductivity of porous medium is considerably higher than fluid thermal conductivity, heat flows horizontally in the porous medium region.

- For the channel with partially porous medium and high $\mathrm{Gr} / \mathrm{Re}$ value, a reverse flow occurs in the channel. The path of heat is also changed accordingly for high Peclet number.

- The use of heatfunction for analyzing heat and fluid flow problems also shows the dimensionless heat transfer rate through the channel. Thus, the increase or decrease of heat transfer through the channel can be predicted.
The present study may be performed for mixed convection in an inclined channel to observe the path of heat flow by changing the inclination angle.

\section{Appendix A}

$$
\begin{aligned}
& C_{1}=\frac{G r_{p}}{\operatorname{Re}}, C_{2}=-\operatorname{csch}(S)\left(\frac{G r_{p}}{2 \operatorname{Re}}+D a \Gamma\right), C_{3}=-\operatorname{csch}(S)\left(\frac{G r_{p}}{2 \operatorname{Re}}-D a \Gamma\right), \\
& C_{4}=-\frac{G r_{p}}{2 \operatorname{Re}}-D a \Gamma, \quad C_{5}=\frac{G r_{c} \theta_{i}}{12 \operatorname{Re}}-\frac{G r_{c}}{12 \operatorname{Re}}, \quad C_{6}=\frac{G r_{c}}{6 \operatorname{Re}}-\frac{G r_{c} \theta_{i}}{6 \operatorname{Re}}+\frac{G r_{c}\left(2 \theta_{i}+1\right)}{12 \operatorname{Re}}, \\
& C_{7}=\frac{G r_{c}\left(2 \theta_{i}+1\right)}{6 \operatorname{Re}} C_{8}=\frac{2 U_{i} S^{2}+2 M \Gamma-2 M \frac{G r_{p}}{\operatorname{Re}} \theta_{i}}{2 S^{2} \sinh (S / 2)}, C_{9}=\frac{M\left(\frac{G r_{p}}{\operatorname{Re}}-2 \Gamma\right)}{2 S^{2} \sinh (S / 2)}, \\
& C_{10}=-\frac{M \frac{G r_{p}}{\operatorname{Re}}\left(-2 \theta_{i}+1\right)}{S^{2}} \quad C_{11}=-\frac{M \Gamma-2 M \frac{G r_{p}}{\operatorname{Re}} \theta_{i}-M \frac{G r_{p}}{2 \operatorname{Re}}}{S^{2}} \\
& C_{12}=1152 M S^{5}-96 M^{2} \frac{G r_{c}}{\operatorname{Re}} S^{3}-144 M^{2} \frac{G r_{p}}{\operatorname{Re}} S^{3}+M \frac{G r_{c}}{\operatorname{Re}} S^{5} \\
& +2304 M^{2} \frac{G r_{p}}{\operatorname{Re}} S+48 M \frac{G r_{p}}{\operatorname{Re}} S^{3}+2 M \frac{G r_{c}}{\operatorname{Re}} \theta_{i} S^{5}-4608 M^{2} \frac{G r_{p}}{\operatorname{Re}} \theta_{i} S \\
& -96 M \frac{G r_{p}}{\operatorname{Re}} \theta_{i} S^{3}+384 M^{2} \frac{G r_{c}}{\operatorname{Re}} \theta_{i} S^{3}-288 M^{2} \frac{G r_{p}}{\operatorname{Re}} \theta_{i} S^{3} \\
& C_{13}=24 M\left(\frac{G r_{c}}{R e} s^{4}-768 M \frac{G r_{p}}{R e}+1536 M \frac{G r_{p}}{R e} \theta_{i}+32 M \frac{G r_{c}}{R e} s^{2}-2 \frac{G r_{c}}{R e} \theta_{i} s^{4}\right. \\
& \left.+4 \frac{G r_{p}}{\operatorname{Re}} \theta_{i} s^{4}-128 M \frac{G r_{c}}{\operatorname{Re}} \theta_{i} s^{2}+192 M \frac{G r_{p}}{\operatorname{Re}} \theta_{i} s^{2}\right)(\sinh (s / 4))^{2} \\
& +2 \frac{G r_{p}}{\operatorname{Re}} \theta_{i} s^{4}-48 M \frac{G r_{p}}{\operatorname{Re}} s^{2}-\frac{G r_{p}}{\operatorname{Re}} s^{4}+96 M \frac{G r_{p}}{\operatorname{Re}} \theta_{i} s^{2} \\
& C_{14}=-1152 \frac{G r_{p}}{\operatorname{Re}} S\left(2 \theta_{i}-1\right) M^{2}, \quad C_{15}=24 S^{6}+2304 M S^{4}-9216 M^{2} S^{2} \\
& C_{16}=-4608 M S^{3}+384 M S^{5}+4608 M^{2} S^{3}, \quad C_{17}=18432 M^{2} s^{2}-2304 M s^{4} \\
& C_{18}=-3 M \frac{G r_{p}}{\operatorname{Re}} S+24 M s^{3}+3 M \frac{G r_{c}}{16 \operatorname{Re}} S^{3}-3 M^{2} \frac{G r_{p}}{\operatorname{Re}} S-5 M \frac{G r_{c}}{8 \operatorname{Re}} \theta_{i} S^{3} \\
& -6 M^{2} \frac{G r_{p}}{\operatorname{Re}} \theta_{i} S+6 M \frac{G r_{p}}{\operatorname{Re}} \theta_{i} S \\
& C_{19}=\cosh (S / 2)\left(\frac{G r_{c} S^{4}}{32 \operatorname{Re}}+12 S^{4}-12 M \frac{G r_{p}}{\operatorname{Re}}+48 M \frac{G r_{p}}{\operatorname{Re}} \theta_{i}+24 M^{2} \frac{G r_{p}}{\operatorname{Re}} \theta_{i}\right. \\
& \left.+M \frac{G r_{c} S^{2}}{2 \operatorname{Re}}-3 M \frac{G r_{p} S^{2}}{2 \operatorname{Re}}-\frac{G r_{c} \theta_{i} S^{4}}{16 \operatorname{Re}}-4 M \frac{G r_{c} \theta_{i} S^{2}}{\operatorname{Re}}-6 M \frac{G r_{p}}{\operatorname{Re}} \theta_{i} S^{2}\right) \\
& +12 M \frac{G r_{p}}{\operatorname{Re}}+24 M^{2} \frac{G r_{p}}{\operatorname{Re}} \sinh (S / 4)^{2}-24 M \frac{G r_{p}}{\operatorname{Re}} \theta_{i}-M \frac{G r_{c} S^{2}}{2 \operatorname{Re}} \\
& +3 M \frac{G r_{p} S^{2}}{2 \operatorname{Re}}+2 M \frac{G r_{c}}{\operatorname{Re}} \theta_{i} S^{2}
\end{aligned}
$$$$
C_{20}=12 M \frac{G r_{p}}{\operatorname{Re}} S \sinh (S / 4)^{2}+6 M \frac{G r_{p}}{\operatorname{Re}} S
$$$$
C_{21}=+12 M S+24 M^{2} \sinh (S / 2)-\frac{S^{4} \sinh (S / 2)}{8}-12 M S^{2} \sinh (S / 2)
$$$$
+24 M S \sinh (S / 4)^{2}
$$$$
C_{22}=-12 M^{2} S-M S^{3}, C_{23}=48 M^{2} \sinh (S / 4)^{2}-24 M^{2}+6 M S^{2}
$$$$
C_{27}=\frac{\left(P, e\left(2 \theta_{i}, C_{10},-, C_{11},+, C_{11}, 2, \theta_{i}\right)\right.}{2} \quad C_{28}=\frac{C_{10} P e\left(-1+2 \theta_{i}\right)}{3}
$$$$
C_{29}=\frac{C_{8} P e\left(1-2 \theta_{i}\right)}{S^{2}} \quad C_{30}=\frac{C_{9} P e\left(1-2 \theta_{i}\right)}{S^{2}}, C_{31}=\frac{2 \theta_{i} C_{8} \mathrm{Pe}}{S},
$$$$
C_{32}=\frac{2 \theta_{i} C_{9} \mathrm{Pe}}{S}, C_{33}=\frac{C_{8} \mathrm{Pe}\left(1-2 \theta_{i}\right)}{S}, C_{34}=\frac{C_{9} \mathrm{Pe}\left(1-2 \theta_{i}\right)}{S}
$$

$C_{35}=\frac{P e\left(2 \theta_{i}+1\right)\left(40 C_{5}+15 C_{6}-6 C_{7}-\frac{5 \Gamma}{2}+80 U_{i}\right)}{960}$ 


$$
\begin{aligned}
C_{36}= & -\left(\frac{\operatorname{Pe}}{S^{2}}\left(\frac{M \Gamma}{8}+\frac{M G r_{p}}{48 \operatorname{Re}}+\frac{M G r_{p} \theta_{i}}{24 \operatorname{Re}}+\frac{3 M \theta_{i} \Gamma}{4}-\frac{7 M G r_{p} \theta_{i}^{2}}{6 \operatorname{Re}}\right)\right) \\
& -\frac{P e}{S^{2} \sinh (S / 2)}\left(\frac { 2 \operatorname { s i n h } ( S / 2 ) } { S ^ { 2 } } \left(\frac{M \Gamma}{2}+\frac{U_{i} S^{2}}{2}-\frac{M G r_{p} \theta_{i}}{2}-M \theta_{i} \Gamma\right.\right. \\
& \left.+\frac{M G r_{p} \theta_{i}^{2}}{\operatorname{Re}}-\theta_{i} U_{i} S^{2}\right)+\frac{2 \theta_{i} \cosh (S / 2)}{S}\left(U_{i} S^{2}+M \Gamma-M \frac{G r_{p}}{\operatorname{Re}} \theta_{i}\right) \\
& -\frac{M\left(2 \theta_{i}-1\right)\left(\frac{G r_{p}}{\operatorname{Re}}-2 \Gamma\right)}{4 S}+\frac{M \theta_{i}\left(\frac{G r_{p}}{\operatorname{Re}}-2 \Gamma\right)}{S} \\
& \left.-\frac{\cosh (S / 2)\left(2 \theta_{i}-1\right)\left(U_{i} S^{2}+M \Gamma-M \frac{G r_{p}}{\operatorname{Re}} \theta_{i}\right)}{2 S}\right)
\end{aligned}
$$

\section{References}

[1] S. Kimura, A. Bejan, The heatline visualization of convective heat transfer, ASME Journal of Heat Transfer 105 (1983) 916-919.

[2] V.A.F. Costa, Unification of the streamline, heatline and massline methods for the visualization of two-dimensional transport phenomena, International Journal of Heat and Mass Transfer 42 (1999) 27-33.

[3] E. Hakyemez, M. Mobedi, H. Oztop, Effects of wall-located heat barrier on conjugate conduction/natural-convection heat transfer and fluid flow in enclosures, Numerical Heat Transfer Part A 54 (2008) 197-220.

[4] M. Mobedi, Conjugate natural convection in a square cavity with finite thickness horizontal walls, International Communications in Heat and Mass Transfer 35 (2008) 503-513.

[5] M. Mobedi, U. Ozkol, B. Sunden, Visualization of diffusion and convection heat transport in a square cavity with natural convection, International Journal of Heat and Mass Transfer 53 (2010) 99-109.
[6] Y. Varol, H.F. Oztop, M. Mobedi, I. Pop, Visualization of heat flow using Bejan's heatline due to natural convection of water near $4{ }^{\circ} \mathrm{C}$ in thick walled porous cavity, International Journal of Heat and Mass Transfer 53 (2010) 1691-1698.

[7] R.S. Kaluri, T. Basak, S. Roy, Bejan's heatlines and numerical visualization of heat flow and thermal mixing in various differentially heated porous square cavities, Numerical Heat Transfer Part A 55 (2009) 487-516.

[8] T. Basak, S. Roy, D. Ramakrishna, I. Pop, Visualization of heat transport during natural convection within porous triangular cavities via heatline approach, Numerical Heat Transfer Part A 57 (2010) 431-452.

[9] R.S. Kaluri, T. Basak, Heatline analysis of thermal mixing due to natural convection in discretely heated porous cavities filled with various fluids, Chemical Engineering Science 65 (2010) 2132-2152.

[10] R.S. Kaluri, R. Anandalakshmi, T. Basak, Bejan's heatline analysis of natura convection in right-angled triangular enclosures: Effects of aspect-ratio and thermal boundary conditions, International Journal of Thermal Sciences 49 (2010) 1576-1592.

[11] M.A. Waheed, Heatfunction formulation of thermal convection in rectangular enclosures filled with porous media, Numerical Heat Transfer Part A 55 (2009) 185-204.

[12] A.I.M. Morega, A. Bejan, Heatline visualization of forced convection laminar boundary layers, International Journal of Heat and Mass Transfer 36 (1993) 3957-3966.

[13] E.M. Sparrow, G.M. Chrysler, L.F. Azevedo, Observed flow reversals and measured-predicted Nusselt numbers for natural convection in a one-sided heated vertical channel, ASME Journal of Heat Transfer 106 (1984) 325-332.

[14] W. Aung, G. Worku, Theory of fully developed, combined including flow reversal, ASME Journal of Heat Transfer 108 (1986) 485-488.

[15] M. Parang, M. Keyhani, Boundary effects in laminar mixed convection flow through an annular porous medium, ASME Journal of Heat Transfer 109 (1987) 1039-1041.

[16] W.J. Chang, W.L. Chang, Mixed convection in a vertical tube partially filled with porous medium, Numerical Heat Transfer Part A 28 (1995) 739-754.

[17] A. Barletta, E. Zanchini, On the choice of the reference temperature for fully-developed mixed convection in a vertical channel, International Journal of Heat and Mass Transfer 42 (1999) 3169-3181.

[18] G. Degan, P. Vasseur, Aiding mixed convection through a vertical anisotropic porous channel with oblique axes, International Journal of Engineering Science 40 (2002) 193-209. 